\title{
Excitation of a Conducting Cylinder Using the Theory of Characteristic Modes
}

\author{
Anita PASCAWATI ${ }^{1}$, Pavel HAZDRA ${ }^{2}$, Tomas LONSKY ${ }^{2}$, Muhammad Reza Kahar AZIZ ${ }^{3}$ \\ ${ }^{1}$ Rocket Technology Center, National Institute of Aeronautics and Space (LAPAN), \\ Raya Lapan Street 2, 16350 Bogor, Indonesia \\ ${ }^{2}$ Dept. of Electromagnetic Field, Czech Technical University in Prague, Technicka 2, 16627 Prague, Czech Republic \\ ${ }^{3}$ Electrical Engineering, Institut Teknologi Sumatera (ITERA), Terusan Ryacudu Street, 35365 Lampung Selatan, Indonesia \\ anita.pascawati@lapan.go.id, \{hazdrap, lonsktom\}@fel.cvut.cz, reza.kahar@el.itera.ac.id
}

Submitted January 1, 2018 / Accepted August 20, 2018

\begin{abstract}
This paper describes the application of the theory of characteristic modes to excite a conducting cylinder representing the chassis of a rocket. Mode excitation is achieved by cutting $H$-shaped slots on the cylinder at specific locations where the maximum of modal current distribution occurs. The L-matching network is designed to match the impedance of the slots to the input coaxial cable. Finally, the proposed concept is verified during manufacturing and measurement. It is shown that the measured results are in excellent agreement with the simulation.
\end{abstract}

\section{Keywords}

Theory of characteristic modes, coupling elements, matching network

\section{Introduction}

The development of antennas for rocket communication systems offers interesting research opportunities in the field of antennas because it is crucial that the transmission of telemetry data from the rocket reaches the ground station. There are several experiments in the designing of antennas for rockets that have been conducted where different types of antennas have been discovered, e.g. printed broadband [1], microstrip [2] and blade [3] antennas. However, it is also essential to ensure that the antenna shape should not disturb the aerodynamics of the rocket [4].

In a rocket application, directional antennas are generally mounted in arrays around the body of a rocket [5] or a wrap-around antenna is used [6] in order to obtain an omnidirectional radiation pattern. Instead of mounting antenna arrays on the rocket, we can use its body as the antenna directly. The inspiration comes from the technology of mobile phones which use the chassis of a printed circuit board (PCB) as the main radiating element [7], [8]. Similarly, the authors use the vehicle body as the main radiator in [9], [10]. To the best of our knowledge, the use of the rocket body as a radiator has not been conducted. Hence, this paper demonstrates the proposed concept of how a rocket chassis (essentially modelled as a perfectly conducting cylinder) can be excited while also acting as an effective radiator. The theory of characteristic modes $(\mathrm{CM})$ is used to find the resonant modes of the rocket chassis [11-15]. Since the modes have orthogonal radiation patterns, several frequencies related to the resonance of the modes can be used simultaneously in the telemetry system of the rocket.

In this paper, the model of the rocket represented as a conducting cylinder has been simulated using CST's CM solver [16] to obtain the characteristic modes. Then the first two modes are studied and excited by utilizing the inductive coupling elements implemented by cutting $\mathrm{H}$-shaped slots on the cylinder. CST's time-domain solver is used for this purpose. Both of the modes, especially the first, are suitable to be applied to the rocket due to a simple current distribution and radiation pattern as well as in the use of fewer slots for excitation. A matching network is designed to match the impedance of the slots to the input $50 \Omega$ coaxial cable. Finally, the measured results of a fabricated structure with the first mode excited shows excellent agreement with the simulation.

\section{The CMs of a Conducting Cylinder}

The theory of characteristic modes was originally developed by Garbacz [11] and later refined by Harrington and Mautz [12]. The method of moments [17] complex impedance matrix $\mathbf{Z}=\mathbf{R}+\mathrm{j} \mathbf{X}$ is decomposed into a set of characteristic currents $\mathbf{J}_{n}$ and characteristic numbers (eigenvalues) $\lambda_{n}$ by solving the following weighted eigenvalue equation [12]

$$
\mathbf{X} \mathbf{J}_{n}=\lambda_{n} \mathbf{R} \mathbf{J}_{n} .
$$

Note that the characteristic currents $\mathbf{J}_{n}$ on the surface of a perfect electric conductor (PEC) body depend only on its shape and frequency, not on any specific excitation. 
The eigenvalues $\lambda_{n}$ represent the ratio of reactive to radiated power [18], and hence, if $\lambda_{n}$ is zero, the $n$-th mode is in resonance. For better dynamics of the resulting graphs, the characteristic angle [14] is usually plotted as

$$
\alpha_{n}=180^{\circ}-\tan ^{-1} \lambda_{n}
$$

where $\alpha_{n}=180^{\circ}$ indicates the resonance of the $n$-th mode. In the CST model, the rocket is modelled as a hollow pipe (cylinder) made of PEC material with length $L=1 \mathrm{~m}$, and diameter $d=0.1 \mathrm{~m}$. Number of surface meshcells in the CST integral solver is around 1500. If the pipe is considered to be a thin dipole, one may guess that the first two resonances are at $f_{1} \cong c_{0} / 2 L=150 \mathrm{MHz}$ and $f_{2} \cong c_{0} / L=300 \mathrm{MHz}$, where $c_{0}$ is the velocity of light in a free space. In this case, where a relatively thick pipe with $L / d=10$ is used, the frequencies are lowered slightly to $129 \mathrm{MHz}$ and $276 \mathrm{MHz}$, respectively, as shown in Fig. 1.

The characteristic currents of PEC cylinder together with their radiation patterns are shown in Fig. 2. The arrows schematically show the direction of the currents and the black rectangles indicate the position of the inductive couplers, which are discussed later. Obviously, the first mode has a sinusoidal in-phase shape, while the second has two opposite-phase currents resulting in two lobes.

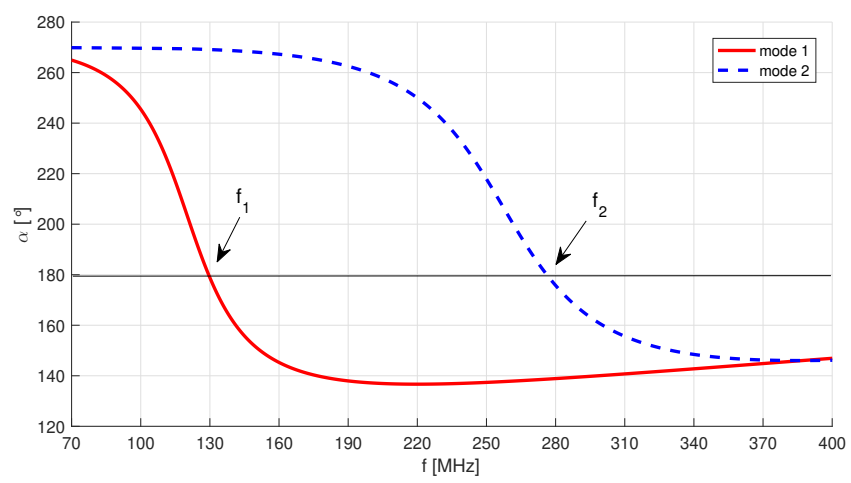

Fig. 1. Variation of the characteristic angle with frequency for the first two characteristic modes of the conducting cylinder.

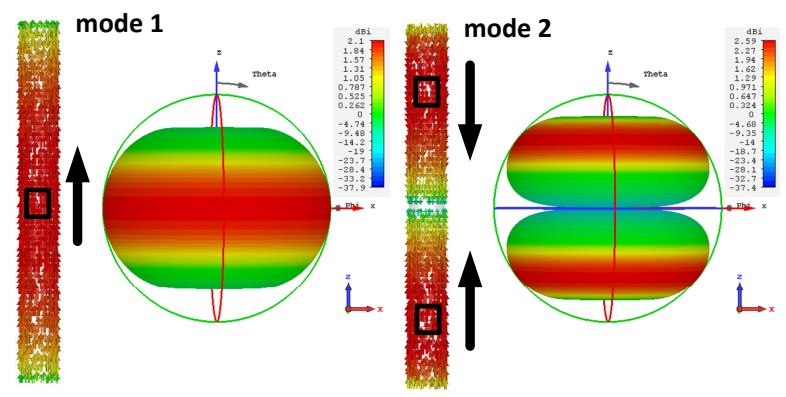

Fig. 2. The first two characteristic currents and directivity patterns at their resonant frequencies $(129 \mathrm{MHz}$ and $276 \mathrm{MHz}$ ) of the conducting cylinder.

\section{Excitation of CMs}

The excitation of modes can be conducted by using inductive coupling elements (ICE). Based on investigations in [7], [8], ICEs are created by cutting small slots at locations where the maximum of characteristic current distributions occur. Therefore, mode 1 can be excited by an inductive coupler at the centre of the cylinder. On the other hand, the characteristic currents of mode 2 flow in opposite directions with two specific maximums. In this case, the mode can be excited by two inductive couplers with a $180^{\circ}$ phase shift. The position of the couplers is schematically shown as black rectangles in Fig. 2.

\subsection{Excitation of CMs using H-Shaped Slots}

To avoid disturbing the distribution of characteristic modes and to maintain the mechanical stability of the design, the ICEs (slots) should be small. In turn, such slots will have low input impedance resulting in problems with matching to $50 \Omega$. In the CST simulations, slots with various shapes were investigated and, based on the results, the H-shaped slot was found to be the preferable solution from an impedance and compactness point of view, see Fig. 3 left. Mode 1 is excited using two in-phase fed $\mathrm{H}$-shaped slots mounted in the middle of the cylinder on its opposite sides (see Fig. 3 right), which helps to obtain a radiation pattern with good azimuthal symmetry.

Mode 2 is excited using two opposite $\mathrm{H}$-shaped slots in the upper part and two opposite H-shaped slots in the lower part of the cylinder. The current distribution and radiation pattern for both properly excited modes is shown in Fig. 4. Calculated maximum directivies are $1.9 \mathrm{dBi}$ and $2.7 \mathrm{dBi}$ for mode 1 and 2, respectively. By comparing with Fig. 2 it is seen that the slots have negligible effect on the far field pattern.

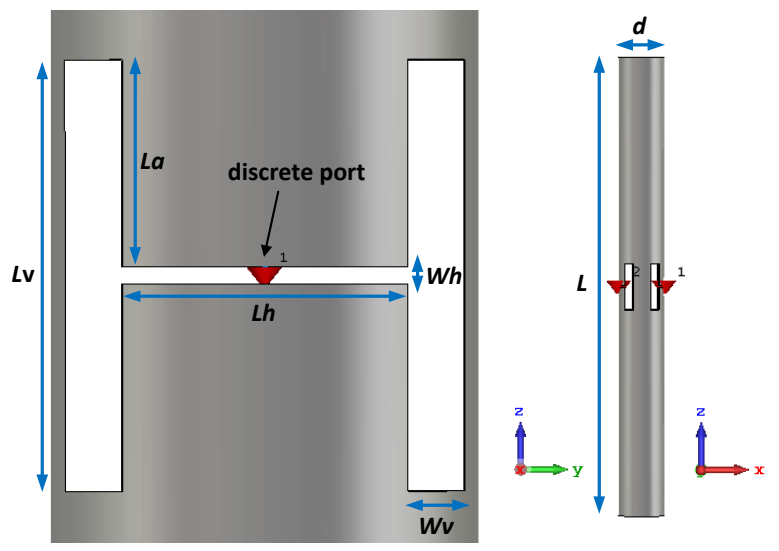

Fig. 3. Dimensions of the H-shaped slot and their location for excitation of mode 1 . 
The excitation mechanism of a modal current is seen in Fig. 5. The currents flowing around the left and right parts of the H-shaped slot have opposite directions and thus do not contribute to the far field. It is dominantly the current in the middle that effectively turns on the mode.

The length and width of the vertical arms of the $\mathrm{H}$-shaped slots are $L_{\mathrm{v}}=100 \mathrm{~mm}$ and $W_{\mathrm{v}}=23.2 \mathrm{~mm}$, those of the horizontal arms are $L_{\mathrm{h}}=72.4 \mathrm{~mm}$ and $W_{\mathrm{h}}=4 \mathrm{~mm}$, and $L_{\mathrm{a}}=48 \mathrm{~mm}$. The longer the length of either $L_{\mathrm{v}}$ or $L_{\mathrm{h}}$, the higher the impedance. The wider the width $W_{\mathrm{v}}$, the higher the impedance as well. In contrast, narrowing $W_{\mathrm{h}}$ results in higher impedance, which explains why $W_{\mathrm{h}}$ is designed to be very thin. Particularly, the impedance of the slots at resonant frequencies of mode 1 and mode 2 are $Z_{\text {mode } 1}=2.69+\mathrm{j} 44.36 \Omega$ and $Z_{\text {mode } 2}=17.39+\mathrm{j} 129.22 \Omega$, respectively. The driven simulation is performed by using the time-domain CST solver with number of mesh cells of around two million.

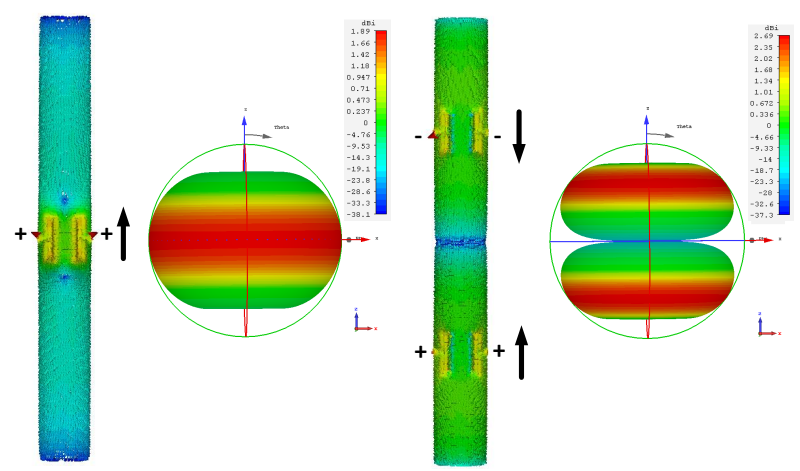

Fig. 4. Current distribution and directivity patterns for excited modes 1 (left) and 2 (right). The arrows schematically show the direction of currents. Mode 1 is excited using two in-phase slots, while mode 2 is excited using two pairs of out-of-phase slots (notice the signs +/- representing the phase of the feeding ports).

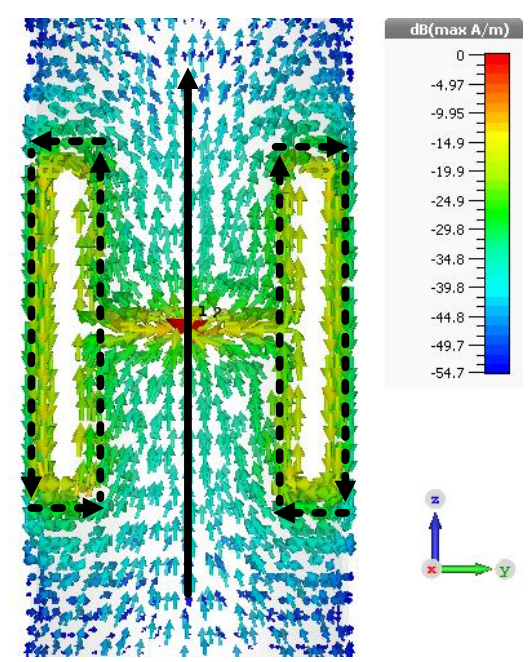

Fig. 5. Dashed arrows represents currents that cancel each other out while the solid arrow is the dominant path that turns on the modal distribution.

\footnotetext{
${ }^{1}$ Resonant frequency is essentialy given by the length of the cylinder.
}

For simplicity of design and ease of manufacturing, we further consider the feeding of only mode 1. By CM analysis, it is confirmed that the slot has very little effect on the resonant frequency of the cylinder ${ }^{1}$, as shown in Fig. 6 where the resonant frequency is $125 \mathrm{MHz}$ with slots, compared to $129 \mathrm{MHz}$ for the whole pipe.

\subsection{Matching the Slots}

Considering the excitation of mode 1, there are two slots which have to be connected in parallel to feed them inphase. There are several possibilities as to how to construct the matching network [19]. In our design we use shunt and series capacitors together with RG58C/U phasing cables as shown in Fig. 7.

The length of the cables was set to $200 \mathrm{~mm}$ and the values of the capacitors were optimized using a schematic tool in CST Studio with the final (rounded) values $C 1=12 \mathrm{pF}$ in series and $C 2=15 \mathrm{pF}$ in parallel. The mechanical arrangement is shown in Fig. 8. For simplicity of construction, we have not used symmetrization like in [20].

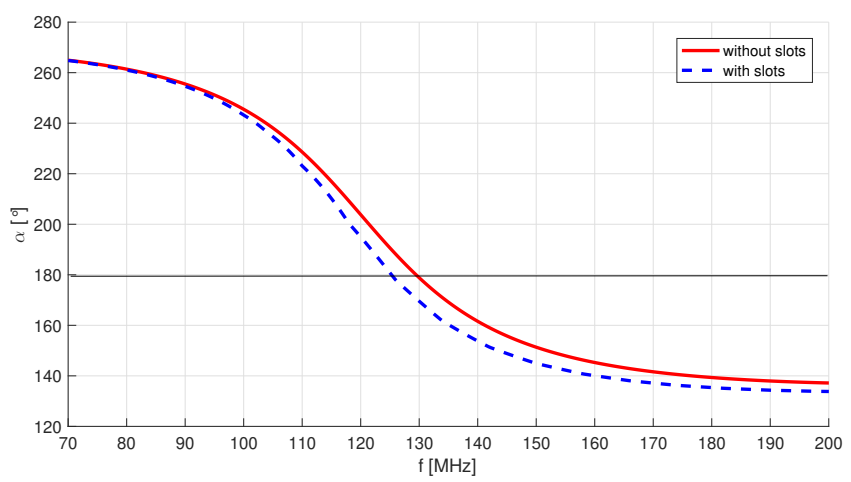

Fig. 6. Characteristic angles of mode 1 with and without slots.

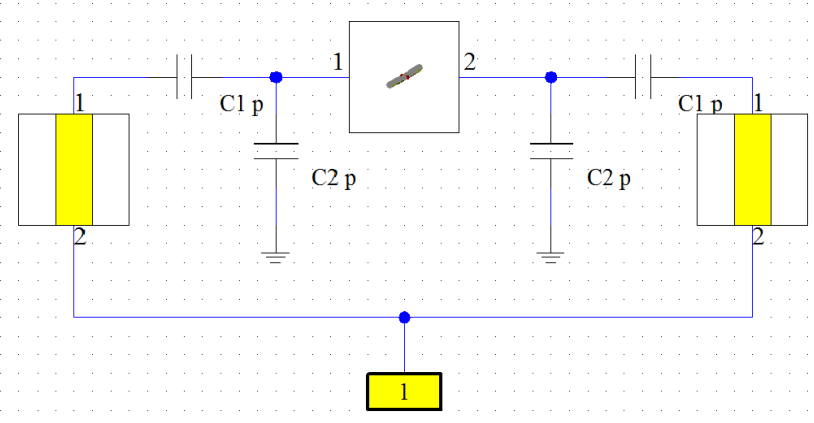

Fig. 7. Matching circuit consisting of shunt and series capacitor and phasing cables (two yellow blocks). The ports 1 and 2 corresponds to that in Fig. 3 right. 


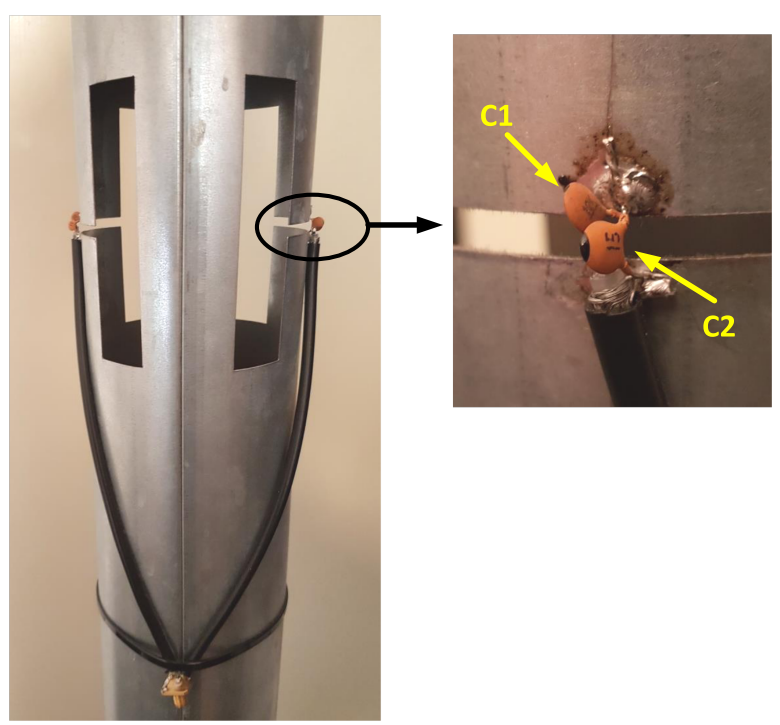

Fig. 8. Detailed view of the matching circuit with capacitors, cables and input SMA connector.

\section{Measurement}

S-parameters at the input SMA connector were measured in an anechoic chamber by the Rohde \& Schwarz ZVA 40 [21] vector network analyzer. Despite the handmade design, the simulated and measured input return loss agree very well, as shown in Fig. 9. In particular, there is a $20 \mathrm{~dB}$ return loss at the resonant frequency of the first mode (125 MHz from Fig. 6). It is noted that there is another small peak around $470 \mathrm{MHz}$, which is likely caused by additional resonance caused by the wires connecting the capacitors.

Unfortunately, the frequency of design is too low to measure the radiation pattern in such a chamber. On the other hand, we had to choose an operating frequency accordingly to the pipe length and diameter routinely found in the market place. Taking into account the agreement between simulated and measured matching, one can also expect agreement for the radiation pattern.

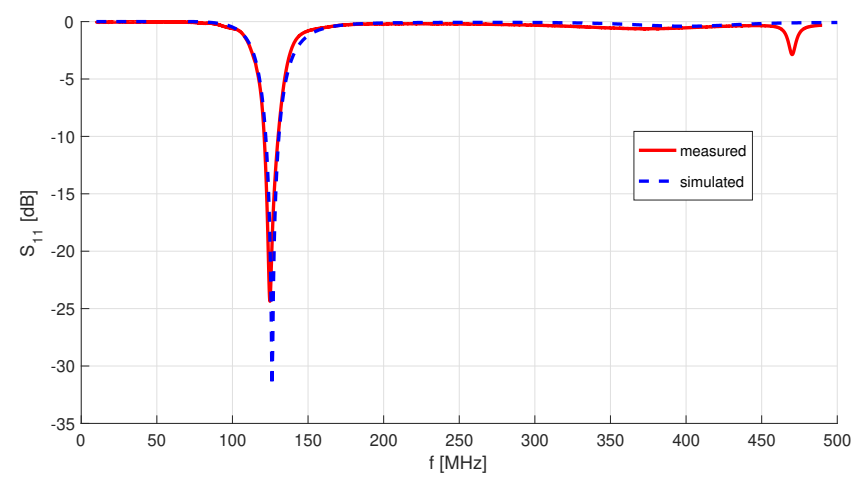

Fig. 9. Simulated and measured S-parameters.

\section{Conclusions}

The excitation of a conducting cylinder using inductive couplers has been presented in this paper. The investigation of the excitation of a rocket chassis as an effective radiator is conducted by the theory of characteristic modes. It was shown that properly designed slots have negligible effect on resonant frequency and current distribution. Although the rocket is modeled in a very simple manner, the presented proof-of-concept indicates that this method can be used in more complex real designs.

\section{Acknowledgments}

This work was supported by the Czech Science Foundation under the project GA17-00607S Complex Electromagnetic Structures and Nanostructures and by the Grant Agency of the Czech Technical University in Prague under the project SGS16/226/OHK3/3T/13 Research on HighFrequency Electromagnetic Structures. It was also supported by Scholarship Program for Research and Innovation in Science and Technology (RISET-Pro) of Kemenristekdikti Indonesia.

\section{References}

[1] PRADES, J., GHIOTTO, A., KERHERVE, E., et al. Broadband sounding rocket antenna for dual-band telemetric and payload data transmission. IEEE Antennas and Wireless Propagation Letters, 2015, vol. 15, p. 540-543. DOI: 10.1109/LAWP.2015.2457338

[2] FILHO, P. C. R., TINOCO-S, A. F., NASCIMENTO, D. C., et al. A telemetry antenna design for a sounding rocket competition. IEEE Antennas and Propagation Magazine, 2017, vol. 59, no. 3, p. 100-140. DOI: 10.1109/MAP.2017.2686096

[3] HE, Y., SEZAI, T., SUNAMI, K. The FDTD analysis of the radiation pattern of an antenna mounted on a rocket. In Proceeding of International Symposium on Antennas and Propagation (ISAP). Okinawa (Japan), 2016, p. 668-669. ISBN: 9784885523137

[4] SRIPHO, P., DUANGSI, S., HONGTHONG, M. Comparison of antenna for dti rocket telemetry system. In Proceedings of the Second Asian Conference on Defence Technology (ACDT). Chiang Mai (Thailand), 2016, p. 105-110. DOI: 10.1109/ACDT.2016.7437652

[5] FILHO, P. C. R., TINOCO-S, A. F., NASCIMENTO, D. C., et al. Flush-mounted telemetry antenna design for a sounding rocket competition. In Proceedings of IEEE Antennas and Propagation Society International Symposium (APSURSI). Orlando (USA), 2013, p. 2173-2174. DOI: 10.1109/APS.2013.6711745

[6] ALBI, R., ZICH, R. E. Wrap-around antenna for rocket telemetry. In Proceedings of IEEE Antennas and Propagation Society International Symposium (APSURSI). Orlando (USA), 2013, p. 1998-1999. DOI: 10.1109/APS.2013.6711657

[7] MARTENS, R., SAFIN, E., MANTEUFFEL, D. Inductive and capacitive excitation of the characteristic modes of small terminals. In Proceedings of Loughborough Antennas \& Propagation Conference (LAPC). Loughborough (UK), 2011, p. 1-4. DOI: 10.1109/LAPC.2011.6114141 
[8] MARTENS, R., SAFIN, E., MANTEUFFEL, D. Selective excitation of characteristic modes on small terminals. In Proceedings of the 5th European Conference on Antennas and Propagation (EUCAP). Roma (Italy), 2011, p. 2492-2496. ISBN: 9788882020743

[9] LI, M., BEHDAD, N. Dual-band platform-mounted HF/VHF antenna design using the characteristic mode theory. IET Microwaves, Antennas \& Propagation, 2018, vol. 12, no. 4, p. 452-458. DOI: 10.1049/iet-map.2017.0612

[10] SHIH, T., BEHDAD, N. Design of vehicle-mounted, compact VHF antennas using characteristic mode theory. In Proceedings of the 11th European Conference on Antennas and Propagation (EUCAP). Paris (France), 2017, p. 1765-1768. DOI: 10.23919/EuCAP.2017.7928334

[11] GARBACZ, R. J., TURPIN R. H. A generalized expansion for radiated and scattered fields. IEEE Transactions on Antennas and Propagation, 1971, vol. 19, no. 3, p. 348-358. DOI: 10.1109/TAP.1971.1139935

[12] HARRINGTON, R. F., MAUTZ, J. R. Theory of characteristic modes for conducting bodies. IEEE Transactions on Antennas and Propagation, 1971, vol. 19, no. 5, p. 622-628. DOI: 10.1109/TAP.1971.1139999

[13] CHEN, Y., WANG, C.-F. Characteristic Modes: Theory and Applications in Antenna Engineering. New Jersey (USA): John Wiley, 2015. ISBN: 9781119038429

[14] CABEDO-FABRES, M., ANTONINO-DAVIU, E., VALERONOGUEIRA, A., et al. The theory of characteristic modes revisited: A contribution to the design of antennas for modern applications. IEEE Antennas and Propagation Magazine, 2007, vol. 49, no. 5, p. 52-68. DOI: 10.1109/MAP.2007.4395295

[15] LAPPALAINEN, J., YLÄ-OIJALA, P., TZAROUCHIS, D., et al. Resonances of characteristic modes for perfectly conducting objects. IEEE Transactions on Antennas and Propagation, 2017, vol. 65 , no. 10, p. 5332-5339. DOI: 10.1109/TAP.2017.2741063

[16] Online. Available at: https://www.cst.com/

[17] HARRINGTON, R. F. Field Computation by Moment Method. New Jersey (USA): John Wiley, 1993. ISBN: 9780780310148

[18] CAPEK, M., HAZDRA, P., MASEK. M., LOSENICKY, V. Analytical representation of characteristic mode decomposition. IEEE Transactions on Antennas and Propagation, 2017, vol. 65, no. 2 , p. 713-720. DOI: 10.1109/TAP.2016.2632725

[19] POZAR, D. M. Microwave Engineering. 4th ed., New Jersey (USA): John Wiley, 2011. ISBN: 9781118213636

[20] LEE, C.-T., SU, S.-W., CHEN, S.-C., FU, C.-S. Low-cost, directfed slot antenna built in metal cover of notebook computer for 2.4-/5.2-/5.8-GHz WLAN operation. IEEE Transactions on Antennas and Propagation, 2017, vol. 65, no. 5, p. 2677-2682. DOI: 10.1109/TAP.2017.2679070

[21] Online. Available at: https://www.rohde-schwarz.com

\section{About the Authors...}

Anita PASCAWATI was born in Bandar Lampung, Indonesia in 1984. She received her bachelor degree (with honors) in Electrical Engineering from the University of Lampung (Unila), Indonesia in 2008 and an M.Sc. in Electrical Engineering from the Czech Technical University in Prague, Czech Republic in 2017. She is currently working in the National Institute of Aeronautics and Space (LAPAN), Indonesia. Her research interests include antenna design for rocket communication and related tracking systems.

Pavel HAZDRA was born in Prague, Czech Republic in 1977. He received his M.Sc. and Ph.D. degree in Electrical Engineering from the Czech Technical University in Prague, in 2003 and 2009, respectively. Since 2012 he has been Associate professor with the Department of Electromagnetic Field at the CTU in Prague. He has authored or coauthored more than 25 journal and 30 conference papers. His research interests are in the area of EM/antenna theory, electrically small antennas, reflector antennas and their feeds and antennas for amater radio purposes.

Tomas LONSKY received his M. Sc. from the Czech Technical University in Prague in 2015. He is working towards his $\mathrm{Ph} . \mathrm{D}$. focused on closely-spaced antenna arrays with use of the Theory of Characteristic Modes. In addition he is developing an antenna arrays for $5 \mathrm{G}$ mobile networks with a direct connection to optical networks.

Muhammad Reza Kahar AZIZ was born in Bandar Lampung, Indonesia in 1981. He received B.Sc. and M.Sc. (cum laude) degrees, in 2004 and 2012, respectively, from Electrical Engineering (Telecommunications), Institut Teknologi Bandung (ITB), Bandung, Indonesia. He received a Ph.D. degree from the Japan Advanced Institute of Science and Technology (JAIST), Ishikawa, Japan, in 2016. In 2004-2005, he was a Microwave Service Engineer with Siemens Indonesia. Dr. Reza has been with Ericsson Indonesia as a Broadband Solution Engineer in 2005-2010. Since 2012, he has joined Institut Teknologi Sumatera (ITERA), Lampung, Indonesia. Dr. Reza received an award as a top $10 \%$ performer in Ericsson Indonesia employee in 2009, ITB Voucher Scholarship in 2011, and JAIST Foundation Research Grant for Students in 2017. He also received Overal Best Paper Award in the Asia Modelling Symposium (AMS), Malaysia, 2015. He has published more than 10 scientific contributions in journals, newsletter, conferences, and scientific speeches. His research interests include wireless communication systems, wireless sensor networks, signal processing, and radio geolocation using factor graph. Currently, he is a member of IEEE and is serving as a reviewer and TPC for IEEE conferences, includes ICC, Globecom, and other international and domestic conferences. He also serves as a reviewer in several journals, includes IEEE TVT, etc. 